Chimia 46 (1992) 111-113

(c) Schweiz. Chemiker-Verband; ISSN 0009-4293

\title{
A Novel Cycloaddition Reaction of Thermally Generated Sulfenes
}

\author{
Ulrich Burger*, Serge P. Schmidlin, Jiri Mareda, and Gerald Bernardinelli
}

Abstract. The highly strained $\gamma$-sultine 4 , resulting from the addition of $\mathrm{SO}_{2}$ to benzobenzvalene, is shown to undergo a thermal cycloreversion which gives $1 \mathrm{H}$ indene-1-thiocarbaldehyde dioxide (6) as an intermediate and subsequently $1 \mathrm{H}$ indene-1-carbaldehyde (5). The sulfene can be intercepted with electron poor $\mathrm{C}=\mathrm{C}$ bonds in a cycloaddition process leading to a five-membered ring. The structure of the fused heterocyclic product $8 \mathrm{~b}$ resulting from addition of the sulfene 6 to $\mathrm{N}$-phenylmaleimide was ascertained by X-ray analysis. Pent-4-enethial dioxide (12) produced by a sulfo-Cope rearrangement from allyl vinyl sulfone (11) is found to add to $N$ phenylmaleimide in the same 1,3-dipolar fashion as the sulfene 6 . The cycloadditions need not be via concerted pathways.

\section{Introduction}

Sulfenes 1 have been invoked, by analogy to ketenes, for many decades as reactive intermediates of the tert-amine-promoted $\mathrm{HCl}$ elimination of $\alpha H$-alkylsulfonyl chlorides or of the complex reaction of $\mathrm{SO}_{2}$ with diazoalkanes. Formal [2+2] cycloadditions with enamines giving aminosubstituted thietane-S,S-dioxides $\mathbf{2}$, or the addition of alcohols providing alkyl sulfonates, were purported to be characteristic sulfene reactions. The well-known formation of mesylates from mesyl chloride, the appropriate alcohol and a tert-amine normally follows this sulfene route rather than proceeding along an $S_{\mathrm{N}} 2$ pathway [1b]. The direct observation of a free sulfene remains elusive despite of the considerable efforts made so far [1]. Recent work suggests that the reactive species resulting from the tert-amine-promoted $\mathrm{HCl}$ elimination of $\alpha H$-alkylsulfonyl chlorides is an amine-stabilized sulfene [2]. In corroboration, several quinuclidine bound sulfenes, e.g. 3 the adduct of bis(trifluoromethyl)sulfene [2c], have been isolated during the eighties with structural determination coming from X-ray crystallography. Some of these amine complexes react with enamines or with enol ethers in the afore-mentioned [2+2] fashion. With

*Correspondence: Prof. U. Burger Department of Organic Chemistry and Laboratory of X-Ray Crystallography University of Geneva $\mathrm{CH}-1211$ Geneva 4 alcohols the amine complexes react to give sulfonates [1c][3].

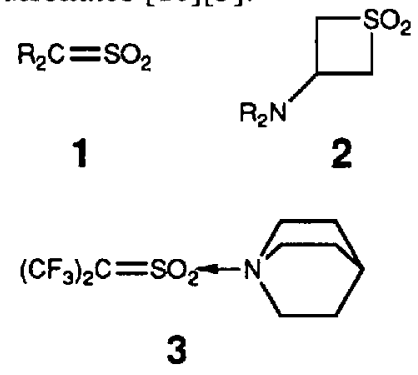

The introduction of a fluorodesilylation method by Block et al. [4] allowed the

Scheme 1 in situ preparation of sulfenes in the absence of tert-amines. The reaction intermediate thus obtained unveiled, in addition to the reactions mentioned above, hitherto unknown dienophilic properties of the sulfenic $\mathrm{C}=\mathrm{S}$ bond. For instance, it undergoes a clean Diels-Alder reaction with cyclopentadiene [4a].

During our studies of thermal and photochemical transformations of strained cyclic sulfinates ('sultines') and of related compounds [5] we became aware of another, presumably genuine, sulfene reaction which to date has not been well studied. It consists of the addition of the reactive intermediate to electron deficient $\pi$ bonds, resulting in the formation of a five membered ring. Overall, the reaction amounts to a cycloaddition with the sulfene being formally a 1,3-dipole.

\section{Results}

In a preliminary communication [5a], we have reported that the highly strained $\gamma$-sultine 4 resulting from the addition of sulfur dioxide to benzobenzvalene, gives $1 H$-indene-1-carbaldehyde (5) and naphthalene, both upon gas-phase pyrolysis (FVP at $380^{\circ}$ ) and upon heating in acid free $\mathrm{CHCl}_{3}$ or $\mathrm{CH}_{2} \mathrm{Cl}_{2}$ (sealed tube, $120^{\circ}$ ) (Scheme 1). To account for this finding, ring opening of 4 was suggested to give the intermediate thiocarbaldehyde dioxide 6 which, upon loss of SO, could give 5 . Extrusion of SO with concomitant formation of a $\mathrm{C}=\mathrm{O}$ function is a known thermal sulfene reaction [6].

The opening of 4 to give 6 , though not necessarily a concerted process, is remi-

\section{Scheme I}

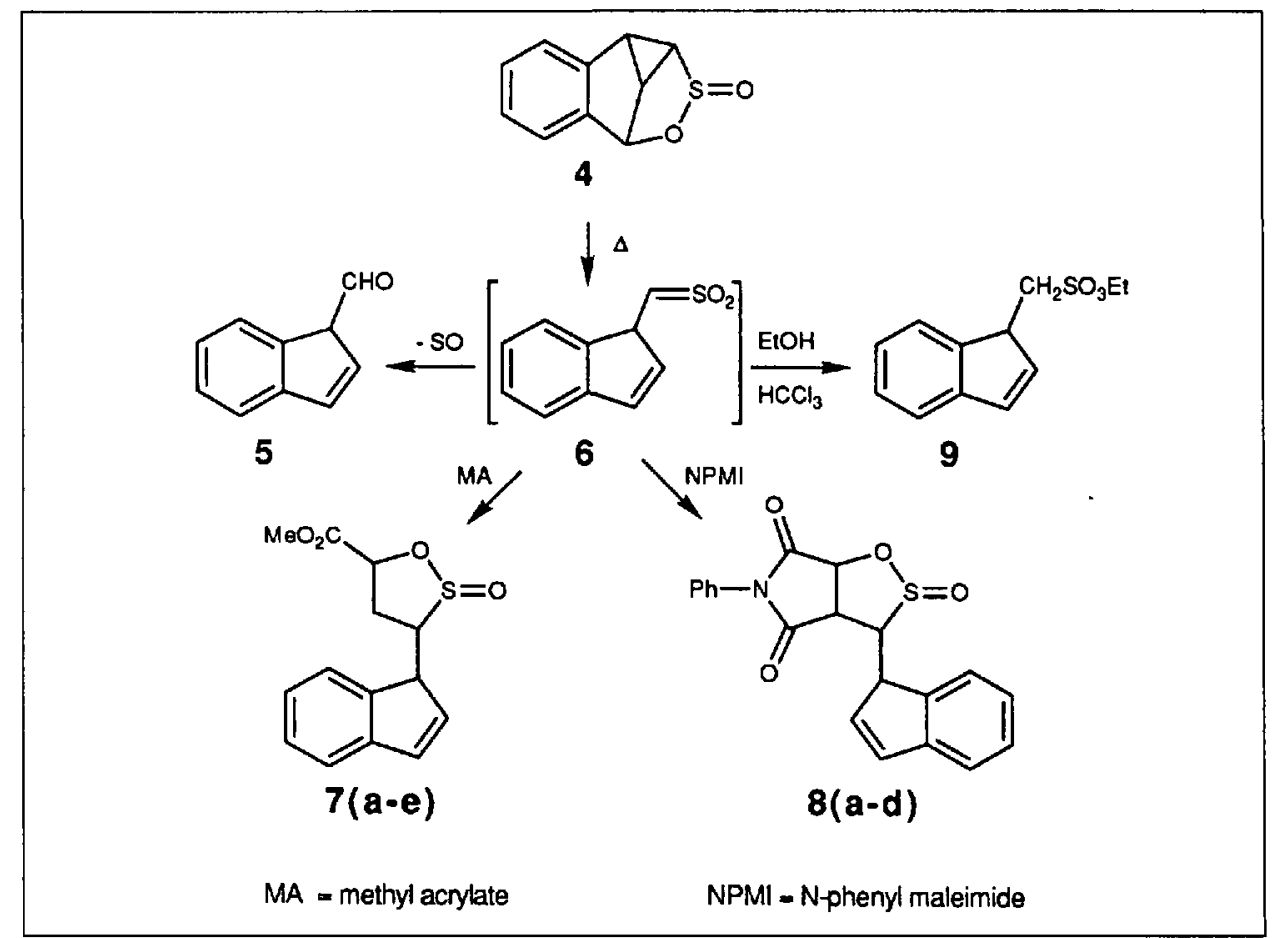




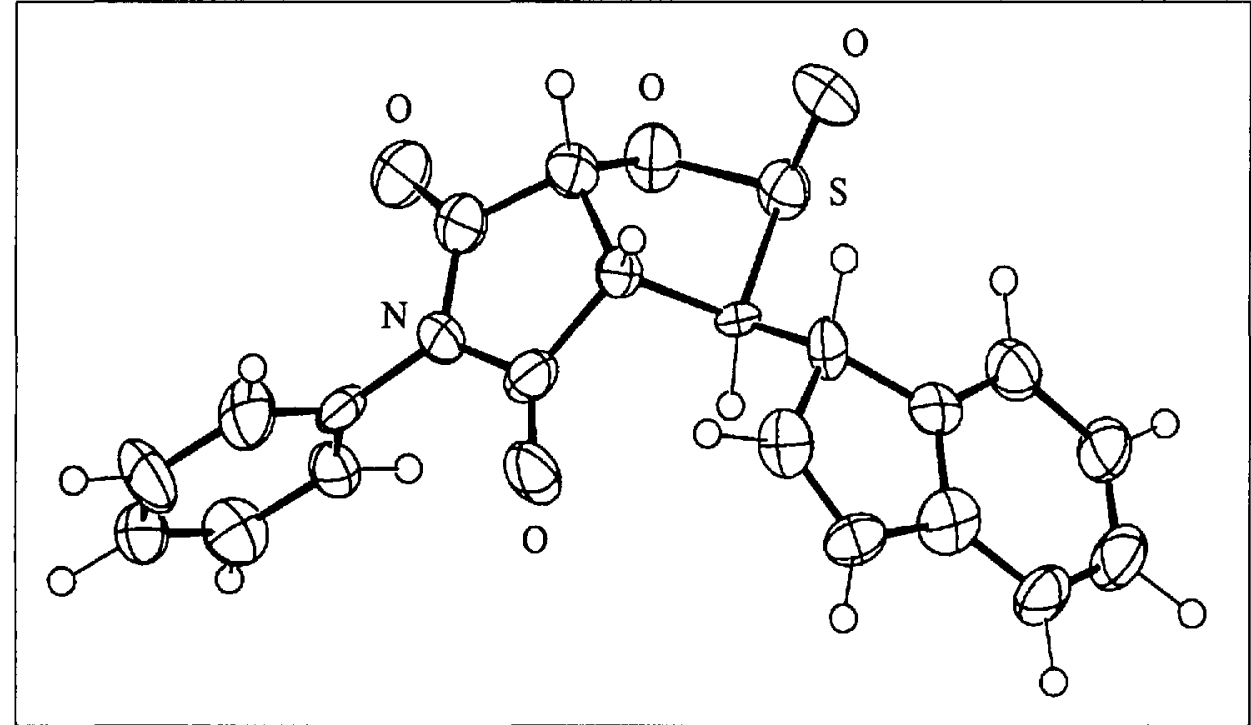

Fig. 1. X-Ray crystal structure of the adduct $\mathbf{8 b}$

niscent of 1,3-dipolar cycloreversion reactions [7]. We attempted to intercept the intermediate 6 with dipolarophiles. Preference was given to $\pi$-electron deficient dipolarophiles due to the electronic nature of sulfenes (vide infra). When we heated the $\gamma$-sultine 4 in $\mathrm{CH}_{2} \mathrm{Cl}_{2}$ with excess methyl acrylate we obtained the stereoisomeric adducts $7(\mathbf{a}-\mathbf{e})$ in $58 \%$ total yield. However, none of them crystallized, and structural proof was limited to standard spectroscopic methods. We have been more successful with $N$-phenylmaleimide (NPMI) as the trapping agent. Four stereoisomeric adducts $\mathbf{8 a - \mathbf { d }}$ were obtained in $82 \%$ total yield (ratio $\mathbf{a} / \mathbf{b} / \mathbf{c} / \mathbf{d}=12: 8: 4: 1$ ). The second most abundant adduct $\mathbf{8 b}$ was readily crystallized (m.p. $189-190^{\circ}$ ), and its structure was determined by $\mathrm{X}$-ray diffraction [8] (Fig. 1). This now provides definitive proof for the formation of the ring system and, in addition, it reveals the relative configuration at the $S$-atom for this particular adduct.

It can clearly be seen in Fig. 1 , that the sulfene moiety has added to $N$-phenylmaleimide with the formation of a fivemembered ring. Both, the indenyl group and the exocyclic oxygen at the S-atom are oriented trans with respect to the N-containing ring.

According to IR, ${ }^{1} \mathrm{H}-\mathrm{NMR}$, and ${ }^{13} \mathrm{C}$ NMR spectroscopy the isomeric adducts $\mathbf{8 a}, \mathbf{8 c}$, and $\mathbf{8 d}$ contain the same fused ring system as $\mathbf{8 b}$, but their precise configuration is unknown at present.

Our hypothesis that the $\gamma$-sultine 4 undergoes a thermal cycloreversion to give the sulfene 6 gains additional support from the trapping reaction with acid-free $\mathrm{EtOH}$. Heating 4 in $\mathrm{CHCl}_{3}$ with excess EtOH (sealed tube, $125^{\circ}, 2 \mathrm{~h}$ ) gives the sulfonate 9 in $87 \%$ isolated yield. It should be mentioned, however, that compound 4 reacts in a different manner with alcohols, if catalytic amounts of acid or base are present. As shown in Scheme 2, ring opening occurs by direct attack at the $\mathrm{S}$-atom of 4 . The reaction was found to be reversible when isolation of the ensuing sulfinate 10 by TLC on silica gel was attempted.

The thermal addition of a sulfene to an electron-deficient $\pi$-system in the previously discussed formal 1,3-dipolar fashion is to our knowledge without precedent, and so far it is an isolated case. The sulfene-type intermediates resulting from the $\mathrm{HCl}$ elimination of $\alpha \mathrm{H}$-alkylsulfonyl chlorides with tert-amines, which is normally performed at room temperature or

Scheme 2

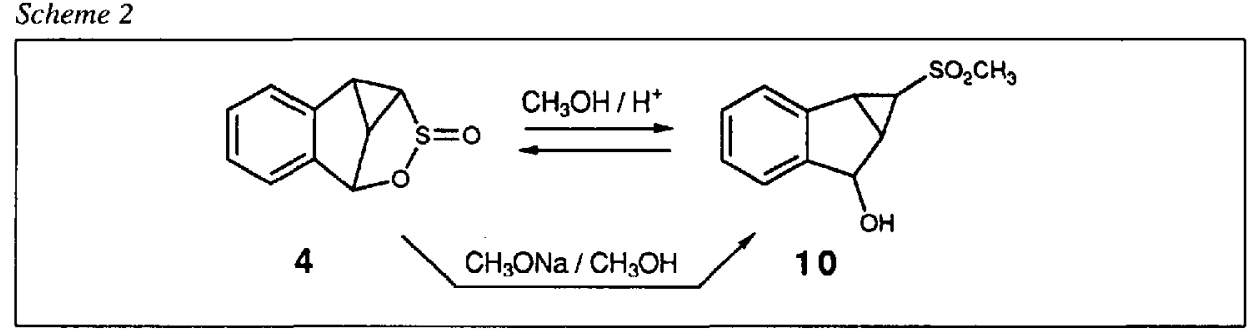

Scheme 3

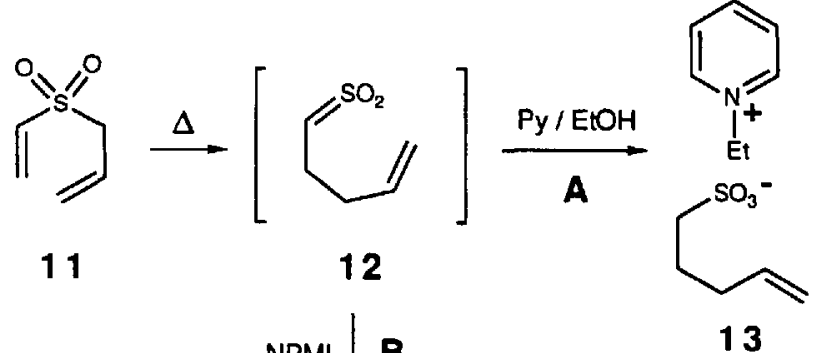<smiles>C=CCCC1C2C(=O)N(c3ccccc3)C(=O)C2OS1=O</smiles>

14 below, are reported not to react with acroleine or acrylates [1c]. Attempts in our laboratory to utilise Blocks fluorodesilylation method [4] to intercept the sulfene with acrylate have not met with success, as dimerization and oligomerization reactions prevail. We, therefore, looked for an alternative method to generate a bona fide free sulfene at high temperature. The [3.3]sigmatropic rearrangement of allyl vinyl sulfone 11 was chosen for that purpose. King et al. [1b][9] have demonstrated that 11 undergoes a sulfo-Cope rearrangement when heated with EtOH/pyridine (sealed tube, $175^{\circ}$ ) to give, via 12, the pyridinium salt 13 (Scheme 3, path $A$ ). When we heated the compound 11 with a fivefold excess of $\mathrm{N}$-phenylmaleimide (sealed tube, $175^{\circ}, 6 \mathrm{~h}$ ), we obtained the adduct 14 in $85 \%$ yield and virtually as a single stereoisomer (Scheme 3, path $B$ ). Compound $\mathbf{1 4}$ which is more sensitive to hydrolytic decomposition than the corresponding $\mathbf{8 b}$ was isolated by mediumpressure chromatography on a short silica-gel column (AcOEt/hexane 1:1) and was fully characterized by standard spectroscopic means. We tentatively assign trans-orientation to the but-3-enyl side chain of 14 with respect to the $\mathrm{N}$-containing ring. The configuration at the $\mathrm{S}$-atom is unknown as yet. Anyway, $\mathbf{1 4}$ has the same fused heterocyclic skeleton as the compounds 8a-d and certainly it has the same mechanistic provenance. 
Table. Total Energies [au], Realtive Energies $\left.{ }^{\mathrm{a}}\right)[\mathrm{kcal} / \mathrm{mol}]$

\begin{tabular}{lllll} 
Molecule & $\begin{array}{l}\text { Point } \\
\text { group }\end{array}$ & $\begin{array}{l}\text { STO-3G* } \\
\text { Total energy }\end{array}$ & $\begin{array}{l}3-21 G^{*} \\
\text { Total energy }\end{array}$ & Relative energy $)^{\mathrm{a}}$ \\
\hline Planar sulfene & $C_{2 v}$ & -579.394046 & -583.252583 & 0.0 \\
Twisted sulfene TS & $C_{2 v}$ & - & -583.177032 & 47.4 \\
Planar methyl sulfene & $C_{s}$ & -617.979813 & -622.075470 & 0.0 \\
Twisted methyl sulfene TS & $C_{1}$ & - & -621.991090 & 53.0
\end{tabular}

a) Relative energies are calculated separately for sulfene and methyl sulfene.

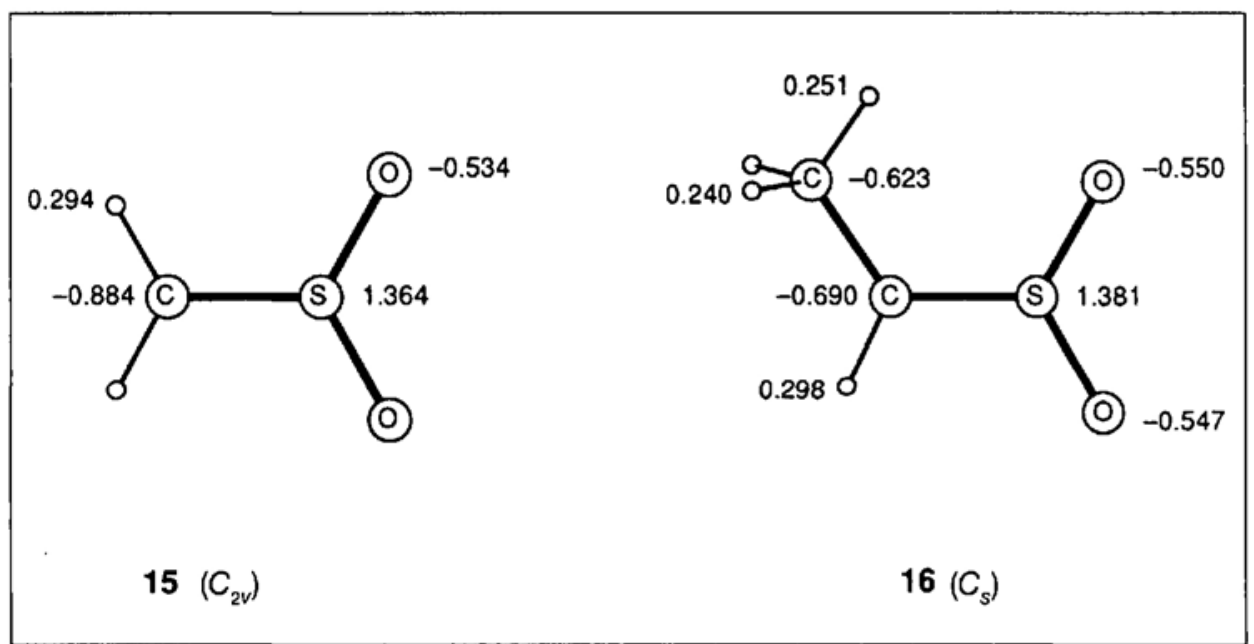

Fig. 2. Perspective drawings of the optimized structures for the planar sulfene $\mathbf{1 5}$ and methyl sulfene 16 together with their atomic charges (3-2IG* basis set)

\section{Discussion and MO Calculations}

The electronic structure of the parent sulfene 15 (thioformaldehyde dioxide; $\mathrm{CH}_{2}=\mathrm{SO}_{2}$ ) has been the object of semiempirical MO calculations at varying degrees of sophistication (CNDO [10a], $\mathrm{CNDO} / 2$ [10b], and CNDO/B [10c]). However, nowadays it is well established that the participation of the d-symmetry functions in the bonding of second-row atoms should be included in the computations [11]. Such polarized functions are capable of reproducing more accurately not only the geometries but also the charge distribution. Therefore, we performed ab initio MO calculations using the GAUSSIAN88 [12] system of programs. Geometries of all structures were optimized at the Hartee-Fock level with the STO-3G* [13] and $3-21 G^{*}[14]$ polarization basis sets. Two stationary points were located on the parent sulfenes potential energy surface, corresponding to the planar $C_{2 v}$ structure and the perpendicular $C_{2 v}$ rotamer. The vibrational frequencies analysis at the 3 $21 \mathrm{G}^{*}$ level permitted the characterization of the former as a global minimum, and the latter as a transition state for the rotation around the $\mathrm{C}-\mathrm{S}$ bond (Table).

To assess the impact of alkyl substitution on the charge distribution in the sulfene, the $a b$ initio computations have also been performed for the methyl sulfene
16 (thioacetaldehyde dioxide). The planar $C_{s}$ structure has been found to be a minimum, whereas the twisted form is a transition state of $C_{1}$ symmetry with the rotational barrier of $53 \mathrm{kcal} / \mathrm{mol}$ (Table). Optimized planar structures of $\mathbf{1 5}$ and $\mathbf{1 6}$ together with the atomic charges are depicted in Fig. 2. For both compounds, it is worthwhile noting, that fairly large positive and negative charges are located on the $\mathrm{S}$ - and the $\mathrm{C}$-atom, respectively.

The addition reactions of the thermally released sulfenes $\mathbf{6}$ and $\mathbf{1 2}$ to the electron poor double bonds of acrylate or maleimide with formation of five-membered rings need not be via concerted cycloadditions. We actually prefer interpretation in terms of a multistep process which starts by conjugate addition of the nucleophilic $\mathrm{C}$ center of the sulfenes to the Michael acceptor.

The very large positive atomic charge on the S-atom accounts for the well-known electrophilic behaviour of sulfenes towards enamines and enol ethers, whereas the large negative charge on the $\mathrm{C}$-atom and its concomitant nucleophilic features provide a good rationale for the cycloaddition described in the present work.

We thank the University of Geneva Computing Center for making available the computing resources of an IBM 3090-180 VF. Financial support was provided by the Swiss National Science Foundation (grant No. 20-27665.89).
Received: January 17, 1992

[1] Reviews: a) B.G. Lenz, B. Zwanenburg, 'Methoden der Org. Chemie HoubenWeyl', 4. Aufl., Thieme, Stuttgart, 1985 ,Bd.E-l 1, Teil 2, S. 1326; b) J.F. King, Acc. Chem. Res. 1975, 8, 10; c) G. Opitz, Angew. Chem. 1967, 79, 161; ibid. Int. Ed. $1967,6,107$.

[2] a) U. Hartwig, H. Pritzkow, W. Sundermeyer, J. Waldi, Z. Naturforsch., B 1988. 43, 271; b) U. Hartwig, H. Pritzkow, W. Sundermeyer, Chem. Ber. 1988, I21, 1435; c) U. Hartwig, H. Pritzkow, K. Rall, W. Sundermeyer, Angew. Chem. 1989, 101, 224; ibid. Int. Ed. 1989, 28, 221; d) H. Pritzkow, K. Rall, S. Reimann-Andersen, W. Sundermeyer, Angew. Chem. 1990, 102, 80; ibid. Int. Ed. 1990, 29, 60.

[3] B.E. Smart, W.J. Middleton, J. Am. Chem. Soc. 1987, 109, 4982.

[4] a) E. Block, M. Aslam, Tetrahedron Lett. 1982, 23, 4202; b) E. Block, A. Wall, $J$. Org. Chem. 1987, 52, 809.

[5] a) U. Burger, D. Erne-Zellweger, A.W. Sledeski, S.P. Schmidlin, Tetrahedron Lett. 1989, 30, 2797; b) U. Burger, C. Gmünder, S.P. Schmidlin, G. Bernardinelli, Helv. Chim. Acta 1990, 73, 1724.

[6] J.F. King, P. De Mayo, C.L. Mclntosh, K. Piers, D.J.H. Smith, Can. J. Chem. 1970, 48, 3707.

[7] Review: G. Bianchi, R. Gandolfi in 'I ,3Dipolar Cycloaddition Chemistry’, Ed. A. Padwa, John Wiley, New York, 1984, Vol. 2, p. 407.

[8] a) G. Bernardinelli, S.P. Schmidlin, U. Burger, submitteld to Z. Kristallogr.; b) Cell parameters and reflection intensities were measured at r.t. on a Nonius-CAD4 diffractometer with graphite monochromated MoK $\alpha$ radiation. The structure was solved by direct methods (MULTAN-87) and refined by least-square analysis with the XTAL programme. Crystallographic data see [8a].

[9] J.F. King, D.R.K. Harding, J. Chem. Soc., Chem. Commun. 1971, 959.

[10] a) J.P. Snyder, J. Org. Chem. 1973, 38, 3967 ; b) K.N. Houk, R.W. Strozier, J.A. Hall, Tetrahedron Lett. 1974, 11,897 ; c) L. Carlsen, J.P. Snyder, J. Org. Chem. 1978, 43, 2216.

[11] For a description of polarization basis sets, see W. J. Hehre, L. Radom, P.v.R. Schleyer, J.A. Pople, 'Ab Initio Molecular Orbital Theory', Wiley-Interscience, New York, 1986.

[12] GAUSSIAN 88, M.J. Frisch, M. HeadGordon, H.B. Schlegel, K. Raghavachari, J.S. Binkley, C. Gonzalez, D.J. DeFrees, D.J. Fox, R.A. Whiteside, R. Seeger, C.F. Melius, J. Baker, R. Martin, L.R. Kahn, J.J.P. Stewart, E.M. Fluder, S. Topiol, and J.A. Pople, Gaussian, Inc., Pittsburgh, PA, 1988.

[13] J.B. Collins, P.v.R. Schleyer, J.S. Binkley, J.A. Pople, J. Chem. Phys, 1976, 64, 5142.

[14] W.J. Pietro, M.M. Francl, W.J. Hehre, D.J. DeFrees, J.A. Pople, J.S. Binkley, J. Am. Chem. Soc. 1982, 104, 5039. 